\title{
Clinical Report \\ Radiological Evaluation of Human Dentin Autografts in Bangladesh
}

\author{
Md. Arafat Kabir'), Masaru Murata'), Koaru Kusano' ${ }^{1)}$,Sayed Mohammad Zakaria'), \\ Abu Hena Mohammad Noor', Fazle Khuda ${ }^{3)}$, Iqbal Hossain²), Shamima Sultana ${ }^{4)}$ and Takashi Saito ${ }^{4)}$ \\ 1) Division of Oral and Maxillofacial Surgery, School of Dentistry, Health Sciences University of Hokkaido, Sapporo, Japan \\ 2) Department of Oral and Maxillofacial Surgery, Pioneer Dental College and Hospital, Dhaka, Bangladesh \\ 3) Japan-Bangla Dental Care, Dhaka, Bangladesh \\ 4) Division of Clinical Cariology and Endodontology, School of Dentistry, Health Sciences University of Hokkaido, Sapporo, Japan \\ (Accepted for publication May 21, 2014)
}

\begin{abstract}
In Dhaka 2013, autografts of demineralized dentin matrix (DDM) were first used as bone regenerative therapy with two patients in Bangladesh. In Case 1, a 29 year-old male presented with an infected periapical lesion involving \#11 and \#12. Root canal treatment followed by periapical surgery were done to remove the lesion. Tooth-derived granules were prepared from a vital non-functional tooth (\#38) by hand-operated crush method, demineralized in $2 \% \mathrm{HNO}_{3}$ for 30 min and washed vigorously. DDM was grafted in the bone defect after the extirpation of the lesion. At 1 year after DDM graft, post-operative radiography indicated excellent bone healing by remodeling with dentin matrix. The pathological diagnosis was radicular granuloma. In Case 2, a 20 year-old female presented with a mesially inclined impacted $3^{\text {rd }}$ molar (\#48). Extraction of the impacted tooth and the autograft of the DDM were done simultaneously. Post-operative radiographs showed the extracted socket healed fully with new bone. The patient-own DDM granules from non-functional teeth could be recycled as osteoinductive materials for local bone regeneration. This works suggest that dentin might become a realistic alternative biomaterial to bone.
\end{abstract}

Key words: Human, Demineralized dentin matrix, Bone regeneration, Autograft

\section{Introduction}

In spite of advancements in field of dentistry, bone regenerative therapy for the repair of bone defect either due to surgical or pathological cause by using osteoinductive biomaterials still remains a daunting task for oral surgeons. Recently, many studies have been performed attempting to accelerate bone repair using different osteoinductive materials. Autogenous bone as a gold standard grafting material has been generally carried out for the regeneration of lost bone. To avoid secondary surgery for harvesting of grafts and morbidity resulting from it, the study for bone substitutes as an alternative of autogenous bone have begun ${ }^{1,2)}$. Therefore, diverse biomaterials have been used in different dental surgery and with continuous research and development as well as clinical advancements, a variety of new biomaterials have been introduced in bone regenerative field. To repair hard and soft tissue defects in conjunction with guided tissue regeneration by grafting materials seems to play a key role not only providing the support to the teeth but also to maintain width and height of alveolar ridge for future prosthesis placement thus Correspondence to: Dr. Masaru Murata, Division of Oral and Maxillofacial Surgery, Health Sciences University of Hokkaido, 1757 Kanazawa, Toubetsu, Ishikari, Hokkaido, 061-0293 Japan; Phone: +81133-23-2921(Direct); E-mail: murata@hoku-iryo-u.ac.jp improving the quality of patient's life ${ }^{1)}$.

In advanced biomaterials sciences, bone inducing materials have a dominant impact on regenerative medicine. Both in medical and dental field there is always a need for biomaterial that allow new bone formation as well as gradual absorption as to be replaced by bone. That's why we have been conducting research on the development of biomaterials using human dentin in co-operation with bone remodeling ${ }^{1}$. While a first clinical report of human bone autograft was done in 1820, human dentin autograft was reported first in IADR 2003 which was a case of sinus lifting using a patient-own DDM granules for bone augmentation ${ }^{4)}$. In 2009, Korea Tooth Bank (KTB) was established in Seoul for the processing of tooth-derived biomaterials which acted as an innovative medical service system making a realistic alternative to bone grafting ${ }^{1,2)}$.

Human tooth is a composite of both organic and inorganic components. Although the structure between dentin and bone are different, both are almost similar in biochemical composition. Dentin and cortical bone consist of body fluid (10\%), collagen (18\%), non-collagenous proteins (NCPs: $2 \%$ ) and hydroxyapatite (HAp: $70 \%$ ) in weight volume. After demineralization, both demineralized dentin matrix (DDM) and demineralized bone 
matrix (DBM) are mainly type I collagen with growth factors such as bone morphogenetic proteins (BMPs) and fibroblast growth factors $(\mathrm{FGFs})^{3,5,7)}$. In addition, due to the limited availability of bone graft substitutes because of their high cost, artificial biomaterials are less popular in developing countries. Based on the previous studies, we focused on the development of a new graft material that promoting bone regeneration.

The aim of this study is to estimate patient-own grafted DDM granules radiologically for regeneration of bone defects after both a periapical surgery and an extraction of wisdom tooth.

\section{Materials and Methods}

\section{Case 1}

\section{Demineralized Dentin autograft with apicoectomy}

A 29-year old male patient reported to the Department of Oral and Maxillofacial Surgery, Pioneer Dental College and Hospital, Dhaka, Bangladesh. He complained a history of discolored upper central anterior teeth with recurrent discharge of pus in relation to the same teeth since 4 to 5 months. Patient had trauma due to bicycle accident in upper anterior teeth in 2006.

\section{Clinical examination}

Clinical examination revealed teeth discoloration along with a slight swelling in the buccal region which was extending from \#11 to \#21 regions. Infected sinus with pus discharge was also observed on the same area. Swelling was soft and fluctuant in nature. Among the upper anterior segment only discolored teeth (\#11 and \#21) were not responsive to the pulp vitality tests, including thermal and electric pulp tester. There were no percussion pain nor any tenderness on the periapical region.

\section{$X$-ray findings and clinical diagnosis}

Periapical dental X-ray and pantomography: Well-defined small rounded radiolucent areas were found attached with the apical part of the traumatized teeth (\#11 and \#21) (Fig. 3a,3b). The apical foramen of the involved teeth was also exposed. This case was diagnosed an infected periapical lesion involving \#11 and \#21. Root canal treatments of \#11 and \#21 were initiated as the first step of treatment planning followed by periapical surgery including apicoectomy with patient-own DDM autograft from vital non-functional wisdom tooth on the next visit. This protocol was approved by the institutional ethics committee and the informed consent was obtained from the patient.

\section{Preparation of autogenous DDM}

His non-functional third molar vital tooth (\#38) was extracted on the 1st visit as a step of our treatment plan. The extracted tooth was kept in the freezer at $-20^{\circ} \mathrm{C}$ until use. DDM was prepared by oral surgeon during the periapical surgery. We used a conventional hand-operated method with order-made stainless steel vessel and a bar. Briefly, frozen tooth was crushed by hammer for $1 \mathrm{~min}$, and interval observation was done to analyze the granule size. The crushed tooth granules were decalcified in $2 \% \mathrm{HNO}_{3}$ solution for $30 \mathrm{~min}$. The DDM granules including cementum were extensively rinsed in cold distilled water (Fig. 1a-1e).

\section{Operative procedure}

Root canal treatment was completed followed by surgical enucleation of cyst, apicoectomy, retrograde filling of involved teeth followed by patient- own demineralized dentin matrix graft. The procedure is as follows: After administration of local anesthesia, incision was given in buccal region which extends from \#13 to \#23 regions. A full thickness mucoperiosteal flap was reflected and irrigated with normal saline. Pathological tissues was removed (Fig. 2a). Root ends of involved teeth were resected and retrograde filling were done. Just before DDM graft, 20 cortical perforations were added to the grafted site, and then DDM were grafted to the bone defect due to the removal of lesion (Fig. $2 b)$. Good wettability of the DDM with blood was observed just after DDM graft (Fig. 2c). Patient was recalled at interval of 1 week, 3, 6 and 12 months. Informed consent was obtained from the patient before image analysis and histological evaluation. The removed tissues were fixed in $10 \%$ formalin solution, dehydrated in an acending series of ethanol, and embedded in paraffin. Thin sections were stained with hematoxylin and eosin (HE) (Fig. 4a, $4 b)$.

\section{Case 2}

\section{Extraction socket regeneration with DDM}

A 20-year old female patient visited to the Department of Oral and Maxillofacial Surgery, Pioneer Dental College and Hospital, Dhaka, Bangladesh. She complained of inability to sustain good oral care at the posterior aspect of the lower right jaw. The chief complains were food impaction in \#47 and \#48 and subsequent malodor. The patient reported non-remarkable medical history.

\section{Clinical examination}

Clinical examination revealed local erytherma with noticeable penetration depth distal to the second molar. There was impacted food debris along with slight pain when pressed to the tissue over the impacted $3^{\text {rd }}$ molar (\#48).

\section{Radiological findings and clinical diagnosis}

Pre-operative periapical X-ray revealed mesially impacted third molar (\#48) in contact with the distal surface of the second molar (\#47) (Fig. 6a) and also there was marked dilation or thickening of the lamina dura secondary due to local inflammatory reaction. After attaining clinical and radiological evaluation, we diagnosed pericoronitis due to the impacted third molar. 
Md. Arafat Kabir et al:: Radiological Evaluation of Human Dentin Autografts in Bangladesh
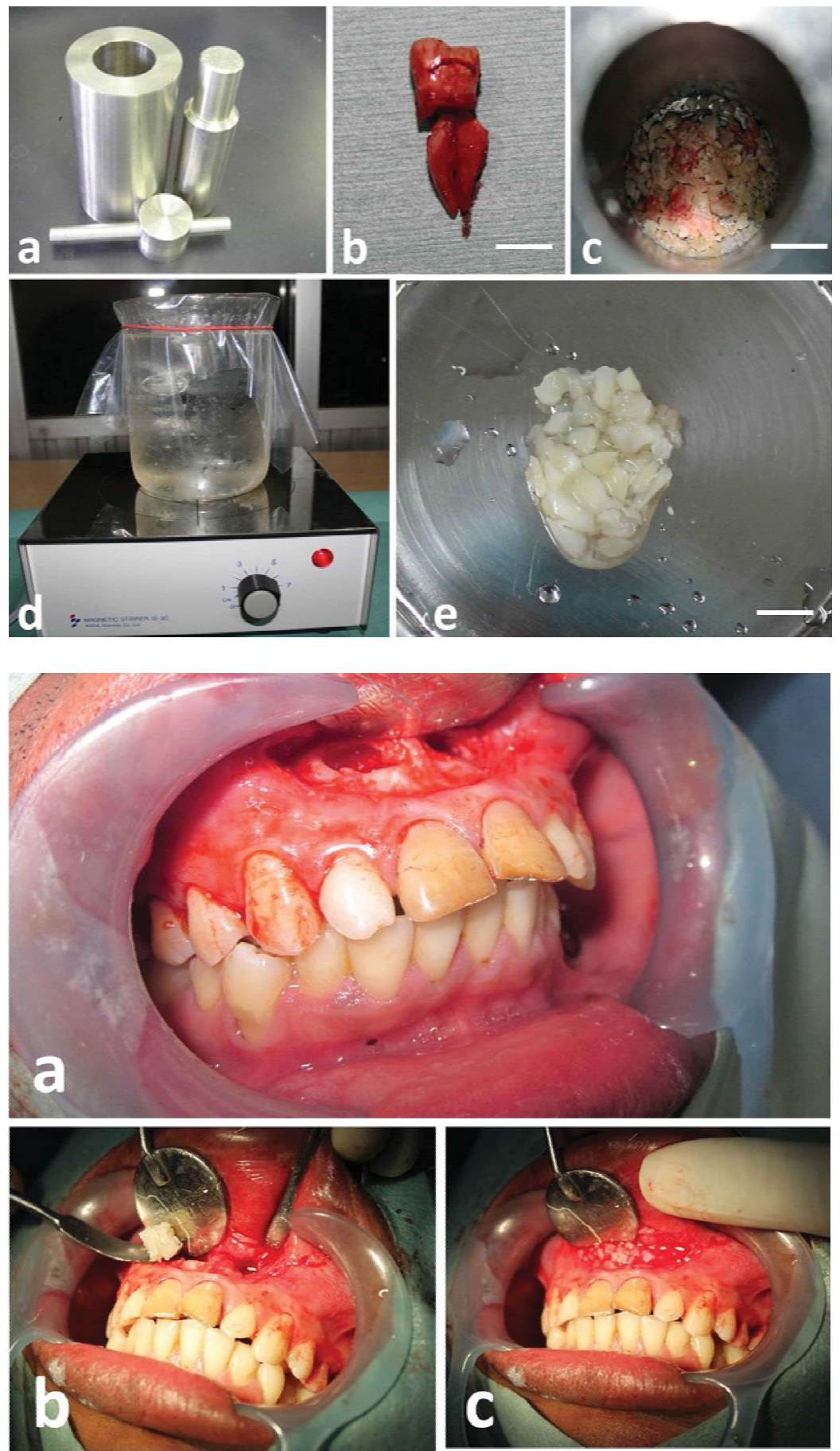

Figure 1. Preparation of DDM in Case 1.

(a) Stainless steel apparatus. (b) Extracted third molar (\#38). (Scale bar $=10 \mathrm{~mm})(\mathrm{c})$ Crushed granules. $(\mathrm{Bar}=5 \mathrm{~mm})(\mathrm{d})$ Demineralization in $2 \% \mathrm{HNO}_{3}$.

(e) Patient-own DDM. (Bar $=2 \mathrm{~mm})$
Figure 2. Photos in DDM autograft.

(a) Bone defects after periapical surgery. (b) Just before DDM graft Note: white DDM. (c) After DDM graft. Note: DDM granules with blood.

\section{Operative procedure}

A mucoperiosteal flap was raised and the bone was exposed under local anesthesia. The third molar was elevated and extracted, followed by debridement and curettage of all debris and granulation tissue of that area. After that we prepared patientown DDM following the same procedure as described in case 1. Just before the grafting of DDM, we made 20 cortical perforations on the socket surface. The volume of DDM was adequate to cover the whole defect (Fig. 5d). Finally, the flap was repositioned and sutured. An immediate post-operative dental X-ray was obtained and the post-operative instructions with medication was given to the patient. The treatment protocol was approved by the institutional ethics committee and informed consent was also obtained from the patient.

These operations and the publication of this clinical report were approved by Pioneer Dental College and Hospital.

\section{Case 1}

\section{Results}

\section{Radiological findings}


J.Hard Tissue Biology Vol. 23(3):363-370, 2014
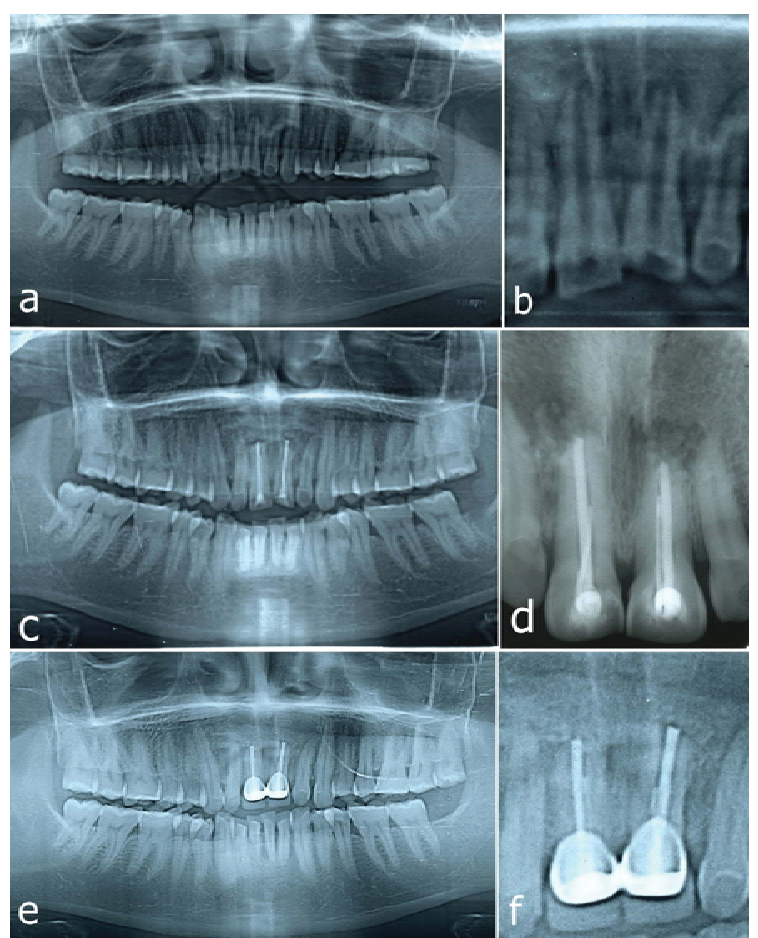

Figure 3. Sequential radiological findings of Case 1.

(a) Pantomograph. (b) X-ray showing radiolucent area related with traumatized teeth (\#11 and \#21).

(c and d) Photo just after DDM graft showed radiopaque particles covering bone defect. (e and f) At 1 year, bone-like radiopaque image in apical defect areas.

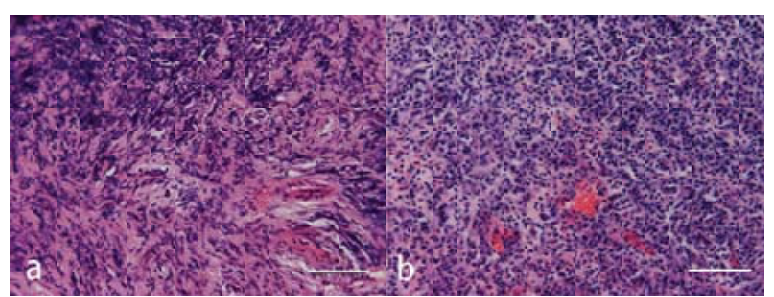

Figure 4. Histological findings of periapical lesion involving \#11 and \#21 in Case 1.

(a) inflammatory fibrous tissue without epithelium. (b) granulation tissue with chronic inflammatory cells infiltration. Note: mainly lymphocytes and no epithelium. (Scale bar $=100 \mu \mathrm{m})(\mathrm{HE})$

Just after the operation, dental X-ray and pantomography showed radiopaque particles in the surgical site (Fig. 3c, 3d) which resembled DDM covering the bone defect except a small radiolucent area close to the resected apical part of \#11, because of unreachability of DDM on that area. At 1 year, radiopaque bone-like tissue was observed covered all parts of defect including that small radiolucent area resulting bone repair (Fig. 4e, 4f).

\section{Histological findings}

HE sections revealed granulation tissue with chronic inflammatory cells composed mainly by lymphocytes, plasma cells and neutrophils (Fig. 4a, 4b). Epithelium was not found in the tissue. The confirmatory diagnosis was a dental granuloma.
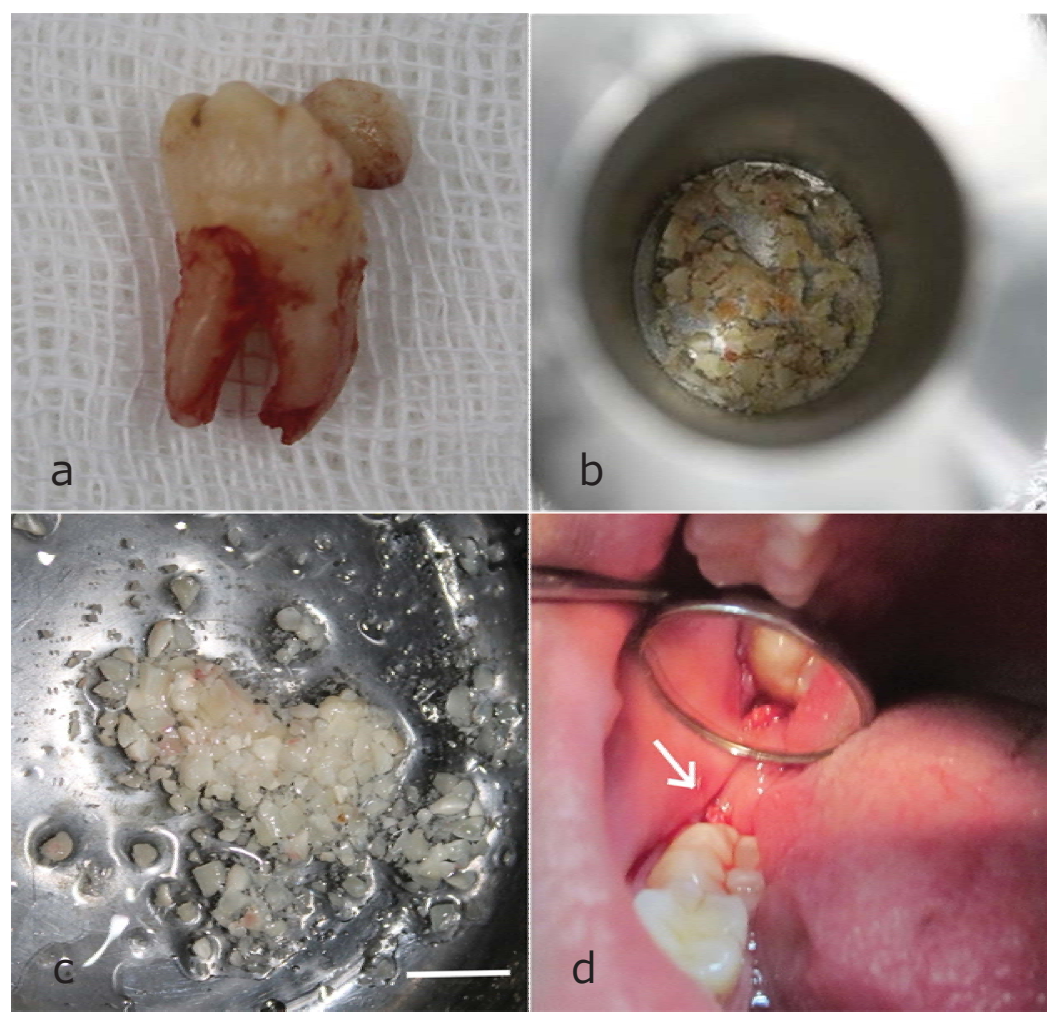

Figure 5. DDM autograft in Case 2.

(a) Extracted vital tooth (\#48). (b) Crushed tooth granules. (c) Dentin matrix (DDM) after demineralization.

(d) Autograft of DDM into extracted socket (Arrow). $(\mathrm{Bar}=10 \mu \mathrm{m})$ 
Md. Arafat Kabir et al.: Radiological Evaluation of Human Dentin Autografts in Bangladesh

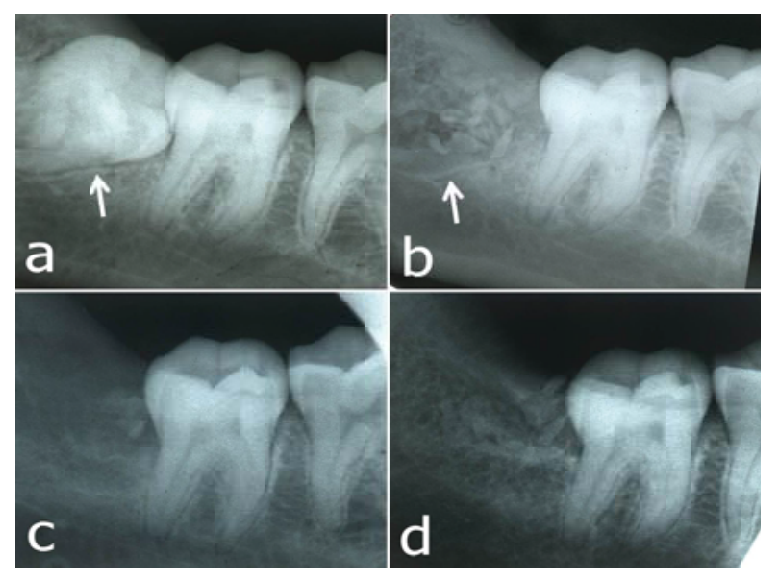

Figure 6. Sequential radiological findings of Case 2.

(a) Preoperative periapical X-ray showing mesially angulated third molar (\#48) contact with the distal surface of the second molar (\#47). Arrow: lamina dura. (b) Just after DDM graft. DDM showing as radiopaque particles inside the extracted socket (\#48). Arrow: lamina dura. (c) At 3 months, almost DDM replaced by bone. Note: small residues of DDM and unclear lamina dura. (d) At 1 year, bone trabecular structure and density similar to the surrounding original bone. Complete disappear of lamina dura showing successful bone healing.

\section{Case 2}

Periapical radiography just after DDM graft revealed radiopaque particles covering fully inside the extracted socket (\#48). The X-ray photo showed the defect was fully grafted with patient-own dentin matrix (Fig. 6b). At 3 months after operation, the quantity of radiopaque particles was visible less than the previous one (Fig. 6c). At 1 year, radiography showed that the extracted socket healed fully with new bone and DDM were absorbed. The photo showed the bone trabecular structure, similar to the surrounding original bone (Fig. 6d). In addition, lamina dura of \#48 disappeared completely by bone remodeling.

\section{Discussion}

Osteoinductive property of rabbit and rat demineralized dentin matrix (DDM) was discovered in $1967^{6}$ ) after the invention of bone-inducing property by rabbit demineralized bone matrix $(\mathrm{DBM})$ in $1965^{3}$. In their rabbit studies, completely demineralized dentin matrix induced bone in the muscle at 4 weeks, while nondemineralized dentin induced scanty amount of bone only during a latent period of 8-12 weeks after implantation. In our previous studies, we confirmed human DDM derived from vital tooth including small patches of cementum and human DBM induced bone and cartilage independently in the subcutaneous tissues of nude mice at 4 weeks ${ }^{8,9}$. These results indicated that osteoinduction was much more earlier in DDM and DBM than calcified dentin and bone. Demineralization technique of dentin and bone increased their osteoinductivity and decrease their antigenesity, while lack of inductive response of the calcified dentin and bone may be related to the restriction of BMPs-release from HAp crystals ${ }^{10,11}$.

After demineralization, both DDM and DBM are composed of predominantly type-I collagen (95\%), non-collagenous proteins (NCPs) including small amount of growth factors ${ }^{12)}$. DDM is defined as an acid-insoluble dentin collagen which is acellular, organic and bioabsorbable matrix with microtube structure as dentinal tubules. DDM can also be defined as acid-insoluble collagen binding bone morphogenetic proteins (BMPs). BMPs were discovered first from bone matrix, and then from animal derived dentin matrix ${ }^{7,13,14)}$. Previous investigations have shown that the dentin matrix proteins have chemotactic, mitogenic, and osteogenic potential. This chemotactic and osteogenic potential of dentin matrix are associated with BMPs and had also reported bone-inducing property in non-skeletal site ${ }^{15)}$. Transforming growth factor-beta (TGF- $\beta$ ), insulin growth factor-I (IGF-I) and IGF-II, fibroblast growth factor (aFGF, bFGF), and platelet derived growth factor (PDGF) were also detected in human dentin, but the concentrations of these growth factors in dentin were lower, compared to bone ${ }^{12)}$.

Bone induction is a basic biological mechanism implies the recruitment of primitive, undifferentiated and pluripotent cells which are stimulated to develop into the bone forming cell lineage ${ }^{16)}$. The principle of bone induction is a complex mechanism of cellular differentiation that depends upon the interaction of inducing cells and responding cells resulting a new pathway of development ${ }^{17}$. It is an important phenomenon which involves the majority of bone healing process. The greater part of newly formed bone depends on the undifferentiated cells that are induced to become bone forming cells, a process which constitutes bone induction. In the mid-1960s, Urist group had used demineralized bone as an osteoinductive agent where he isolated a soluble glycoprotein called BMP as the inductive agent. The history of bone morphogenetic proteins involved in dentin with the reports in 1967 that rabbit DDM induced bone formation in the intramascular pockets, while in 1990 BMPs were detected in human dentin ${ }^{6,12,13)}$. It is well known that BMP-2 is the principle inductive agent for bone and cartilage.

The process of bone induction by DDM is the same way as like DBM. Osteogenesis occur in two different manners, intramembranous ossification or endochondral ossification. We reported human DDM induced bone and cartilage independently at 4 weeks after implantation ${ }^{5}$. Although there are some microenvironmental variables such as surface of dentin matrix, rate of $\mathrm{O}_{2}$ saturation, $\mathrm{CO}_{2}$ tension, other cell metabolic products, Bassett has reported about the influence of $\mathrm{O}_{2}$ concentration on differentiation of connective tissue in $1961^{18)}$. It is mentioned that high $\mathrm{O}_{2}$ concentration favors direct bone formation while low $\mathrm{O}_{2}$ concentration cause endochondral ossification ${ }^{18,19)}$. From above discussion, it can be state that DDM is a bioabsorbable, acidinsoluble collagen matrix and gradually absorbed to be replaced 
and remodeled by new bone.

Although many bone graft substitutes are commercially available as alternatives of autogenous bone in the developed countries, bone autograft in the developing countries including Bangladesh is still the most popular option for bone regeneration. Artificial biomaterials are not much popular in the developing countries, because of their high cost. Autogenous bone graft has some drawbacks like a need of secondary surgery and a donor site morbidity. Autogenous dentin graft can be regarded as an innovative graft material owing to its similar composition and osteoinduction, osteoconduction and osteointregation properties like bone. Considering these facts, we achieved a revolutionary step to introduce DDM as an alternative to bone graft in Bangladesh, which became the 1 st clinical dentin autograft. In our study, the results of radiological findings showed that $90 \%$ $95 \%$ of patient-own recycled dentin matrix had remodeled into bone resulting excellent bone defect repair thus opening an unique way of treatment in Bangladesh dentistry.

In this study, DDM was used as a collagenous biomaterial for bone regeneration. In both cases, patients were successfully received DDM graft without any complexity. Bacteria-free of the DDM was confirmed by the bacteriological examination before use from our previous study ${ }^{20)}$. The grafted DDM was followed by X-ray until 12 months, and both patients have been clinically asymptomatic until now. In addition, the gum and mucosa healed very well in both cases. The DDM granules were received well to hosts. Radiographic evaluation showed noticeable enhancement of density and radiopacity on the grafted site resulting complete bony repair by new bone formation from patient-own DDM. In case 1 , small radiolucent area due to unavailability of DDM during grafting was also recovered and refilled with new bone, resulting in successful bone healing. The DDM residues were gradually absorbed and remodeled into bone during 12 months after graft. In case 2, DDM residues were seen near the enamel of second molar (\#47) (Fig. 6d). The area was considered to be difficult for DDM to transform into new bone due to lack of inducing source. When the socket was filled with DDM, a sharp radiopaque layer of lamina dura was observed just after grafting (Fig. 6b). At 3 months, the quantity of radiopaque particles were visible less than the previous one (Fig. 6b, 6c) Gradual absorption of DDM and disappear of lamina dura indicated successful remodeling of DDM into new bone (Fig. 6c). Eventually, DDM contributed to the height maintenance of alveolar ridge. Our procedure for the preparation of DDM enabled to achieve immediate dentin autograft. The clinical and radiographic results demonstrated that the transplant of DDM into bone defects was a good treatment option for bone regeneration. We concluded that patient-own DDM granules from non-functional teeth should be recycled as osteoinductive materials for local bone regeneration, and dentin could become a realistic alternative material to bone graft.

\section{References}

1. Murata M, Akazawa T, Mitsugi M, Kabir MA, UmIW,Minamida Y, Kim KW, Kim YK, Sun Y and Qin C. Autograft of dentin materials for bone regeneration. In: Advances in Biomaterials Science and Biomedical Applications, ed by Pignatello R, InTech publisher, Croatia, 2013, pp 391-403

2. Murata M, Akazawa T, Mitsugi M, Um IW, Kim KW and Kim YK. Human dentin as novel biomaterial for bone regeneration. In: Biomaterials - Physics and Chemistry, ed by Pignatello R, InTech publisher, Croatia, 2011, pp 127140

3. Urist MR. Bone: formation by autoinduction. Science 150 : 893-899, 1965

4. Murata M. Autogenous demineralized dentin matrix for maxillary sinus augmentationin in human. The first clinical report. J Dent Res 82: B-243, 2003

5. Murata M, Kawai T, Kawakami T, Akazawa T, Tazaki J, Ito K, Kusano K, and Arisue M. Human acid-insoluable dentin with BMP-2 accelerates bone induction in subcutaneous and intramascular tissues. J Ceram Soc Jpn 118: 438-441, 2010

6. Bang $\mathrm{G}$ and Urist MR. Bone induction in excavation chambers in matrix of decalcified dentin. Arch Surg 94: 781-789, 1967

7. Butler WT, Mikulski A, Urist MR, Bridges G and Uyeno S. Noncollagenous proteins of a rat dentin matrix possessing bone morphogenetic activity. J Dent Res 56: 228-232, 1977

8. Murata M, Akazawa T, Takahata M, Ito M, Tazaki J, Hino J, Nakamura K, Iwasaki N, Shibata T and Arisue M. Bone induction of human tooth and bone crushed by newly developed automatic mill. J Ceram Soc Jpn 118: 434-437, 2010

9. Murata M, Akazawa T, Tazaki J, Ito K, Hino J, Kamuira Y, Kumazawa R, and Arisue M. Human dentin autograft for bone regeneration -automatic pulverizing machine and biopsy- . Bioceramics 22: 745-748, 2009

10. Reddi AH. Bone matrix in the solid state: geometric influence on differentiation of fibroblasts. Adv Biol Med Phys 15: 118,1974

11. Huggins CB, Wiseman S and Reddi AH. Transformation of fibroblasts by allogeneic and xenogeneic transplants of demineralized tooth and bone. J Exp Med 132: 1250-1258, 1970

12. Finkelman RD, Mohan S, Jennings JC, Taylor AK, Jepsen S and Baylink DJ. Quantitation of growth factors IGF-I, SGF/ IGF-II, and TGF-beta in human dentin. J Bone Miner Res 5, 7: 717-723, 1990

13. Bessho K, Tagawa T and Murata M. Purification of rabbit bone morphogenetic protein derived from bone, dentin, and wound tissue after tooth extraction. J Oral Maxillofac Surg 48: $162-169,1990$ 
Md. Arafat Kabir et al.: Radiological Evaluation of Human Dentin Autografts in Bangladesh

14. Kawai T and Urist MR. Bovine tooth-derived bone morphogenetic protein. J Dent Res 68: 1069-1074, 1989

15. Murata M, Inoue M, Arisue M, Kuboki Y and Nagai N. Carrier-dependency of cellular differentiation induced by bone morphogenetic protein (BMP) in ectopic sites. Int J Oral Maxillofac Surg 27: 391-396, 1998

16. Albrektsson $\mathrm{T}$ and Johansson C. Osteoinduction, osteoconduction and osseointegration. Eur Spine J 10: 96101,2001

17. Yeomans JD and Urist MR. Bone induction by demineralized dentine implanted into the oral, osseous, and muscle tissues. Arch Oral Biol 12: 999-1008, 1969
18. Bassett CAL. Influence of oxygen concentration and mechanical factors on differentiation of connective tissues in vitro. Nature 190: 460-461, 1961

19. Brighton CT and Heppenstall RB. Oxygen tension in zones of the epiphyseal plate, metaphysis and diaphysis. J Bone Joint Surg 53A: 719-728, 1971

20. Fujita M, Uehara O, Miyakawa H, Naito S, Kabir MA, Murata $\mathrm{M}$ and Nakazawa F. Detection of bacteria in human tooth-derived biomaterials. In: Advances in Oral Tissue Enginering, ed by Murata M and Um IW, Quintessence Publishing Co, Inc., Chicago, 2014, pp 31-32. 
J.Hard Tissue Biology Vol. 23(3):363-370, 2014 